\title{
Gestantes adolescentes en la perspectiva de tres docentes de educación física escolar
}

Jhon Carlos Cortés Murillo

Magister en Estudios Sociales. Corporación Universitaria CENDA - Colombia

Jhon.cortes@cenda.edu.co https://orcid.org/oooo-0001-8160-7979

\section{Ita del Pilar Perea Baena}

Magister en administración de empresas.

Corporación Universitaria CENDA

- Colombia

https://orcid.org/oooo-0oo2- 4496-9863

\section{Artículo de Investigación}

Recepción: 12 de febrero de 2019

Aprobación: 30 de mayo de 2019 https://doi.org/10.19053/22160159.v10.n25.2019.8230

\section{Resumen}

Esta investigación exploratoria de tipo cualitativo busca interpretar el sentir-pensarhacer de tres maestros de educación física a partir de su conocimiento y decisiones frente a casos de estudiantes en estado de gestación. Los sentimientos, conocimientos y actuaciones del maestro se despliegan en actividades y se reflejan en las percepciones de sus estudiantes, lo cual genera en ellos modos de pensar, comprender y significar los procesos de la clase. Para el trabajo de campo se realizaron observaciones no participantes en ficha de registro tres veces por semana durante un periodo de cinco meses. Como complemento, se desarrollaron seis entrevistas semiestructuradas de dos encuentros con cada participante. Los resultados indican que los docentes actúan de acuerdo con los imperativos disciplinares que privilegian el cuidado del cuerpo biofísico por encima de los 
aspectos socioculturales, razón por la cual han hecho del desempeño físico y desarrollo motor los ejes de sus propuestas educativas.

Palabras clave: educación física, sentir, pensar, hacer, madres gestantes

\section{Young pregnant women from the perspective of three school physical education teachers}

\section{Abstract}

This qualitative exploratory study seeks to interpret the feelings, thoughts, and actions of three physical education teachers, based on their knowledge and decisions regarding cases of pregnant students. Teachers' feelings, knowledge, and actions are found in activities and they are reflected in the students' perceptions, which encourage them to think, understand, and give meaning to the class processes. The fieldwork included six non-participant observations described in a record sheet three times a week for five months. As a complement to this exercise, six semi-structured interviews were carried out based on two meetings with each participant. The results suggest that teachers act in accordance with disciplinary imperatives that privilege biophysical body care beyond sociocultural aspects, which is why they have made physical performance and motor development the main lines of their educational proposals.

Keywords: physical education, feeling, thinking, making, expectant mothers

\section{Jeunes femmes enceintes du point de vue de trois enseignants d'éducation physique scolaire}

\section{Résumé}

Cette recherche exploratoire de type qualitative vise à interpréter les sentiments, la pensée, et les actions de trois enseignants d'éducation physique et sportive, à partir de leur connaissance et leurs décisions concernant des étudiantes en état de grossesse. Les sentiments, les connaissances, et les actions des enseignants se 
déploient dans des activités et sont reflétés dans la perception des étudiants, ce qui les encourage à penser, comprendre, et donner une signification aux processus de la classe. Des observations non-participantes ont été réalisées et énoncées dans une fiche d'enregistrement trois fois par semaine pendant cinq mois. Comme complément à cet exercice, six entretiens semi-structurés ont été développés à partir de deux rencontres avec chaque participant. Les résultats indiquent que les enseignants agissent conformément aux impératifs disciplinaires privilégiant le soin du corps biophysique au-delà des aspects socioculturels, raison pour laquelle ils ont fait de la performance physique et du développement moteur les axes de leurs propositions éducatives.

Mots-clés : éducation physique, sentir, penser, faire, femmes enceintes

\section{Gestantes adolescentes na perspectiva de três docentes de educação física escolar}

\section{Resumo}

Esta pesquisa exploratória de tipo qualitativo procura interpretar o sentir-pensar-fazer de três maestros de educação física a partir de seu conhecimento e decisões em frente a casos de estudantes em estado de gestação. Os sentimentos, conhecimentos e atuações do maestro se desdobram em atividades e refletem-se nas percepções de seus estudantes, o qual gera neles modos de pensar, compreender e significar os processos da classe. Para o trabalho de campo se realizaram observações não participantes em ficha de registro três vezes por semana durante um período de cinco meses. Como complemento a este exercício, se desenvolveram seis entrevistas semiestruturadas de dois encontros com cada participante. Os resultados indicam que os docentes atuam de acordo com os imperativos disciplinares que privilegiam o cuidado do corpo biofísico acima dos aspectos socioculturais, razão pela qual têm feito do desempenho físico e desenvolvimento motor os eixos de suas propostas educativas.

Palavras-chave: educação física, sentir, pensar, fazer, mães gestantes 


\section{Introducción}

La complejidad de la realidad cotidiana de las clases de educación física escolar se configura en razón del conjunto de situaciones y condiciones que van emergiendo y de sus estructuras constantes. Sin embargo, realidades externas como la familia, los amigos, el amor y otros tantos mundos del estudiante inciden en los actores y actuaciones de la escuela. Así, la educación física, al igual que otras asignaturas escolares, debe hacer frente a tales situaciones, dado que la vida cotidiana escolar se traslapa con los demás escenarios de la vida y se afectan recíprocamente.

El imperativo de formación escolar que pretende marcar la vida de los estudiantes como un modelo predeterminado y lineal olvida reconocer la importancia educativa del sentir, pensar y hacer en una triada que los mantiene fundidos a los cuerpos de aquellos que constituyen los ambientes de aprendizaje, a saber, estudiantes y maestros. "En el acto de conocer la realidad, los pensamientos y acciones están entrelazados con las emociones y los sentimientos, con los deseos y afectos, generando una dinámica procesal que expresa la totalidad humana" (Moraes \& Torre, 2002, p. 47). Bajo estas circunstancias, es clave preguntar ¿̇cómo podemos conocer al sujeto desde sus acciones sin caer en los determinismos conductistas y segmentarios? Para esto será fundamental comprender que la vida escolar está envuelta en procesos autoorganizativos, de autoconstrucción, en los que el cuerpo y sus acciones están más allá de las estructuras materiales, pues este también se configura en lo emocional, social, histórico y contextual. De acuerdo con Torre (2011), se trata de un proceso mediante el cual se articula el pensamiento y el sentimiento hasta converger en un acto de conocimiento, en un proceso de fusión y de integración. Según el autor, es una concepción holística de la realidad educativa, donde lo biológico, neurológico, psicológico y sociocultural representan dimensiones parciales de una misma realidad compleja que se encuentra plenamente integrada en la vida misma.

Si la escuela pretende educar en la integralidad humana; los pensamientos, emociones, intuiciones, sentimientos y acciones de la subjetividad deben dialogar constantemente con los contenidos de la razón objetiva, es decir, es necesario que las teorías educativas emprendan la exploración de nuevas cuestiones epistemológicas 
que van más allá de llenar los espacios de aprendizaje con un conjunto de elaboraciones conceptuales importadas de las distintas disciplinas o ciencias, lo cual no significa desestimarlas, pues en ellas existe la posibilidad de configurar escenarios que vinculan las pasiones, el placer, la emotividad, el gusto, los sentimientos y otras formas expresivas, con el saber, la razón y lo metódico; esto sin que la fuerza epistemológica y metodológica de cada área de conocimiento quede condicionada, limitada y por fuera de la vida escolar, humana y cotidiana. Para Maturana y Varela (1999) lo humano comprende los aspectos relacionados con el dominio físicobiológico, pero también comprende un dominio simbólico que surge con el pensamiento, la conciencia y el lenguaje humano, aspectos en los cuales lo emocional, la toma de decisiones y las formas de actuar constituyen redes de acciones coordinadas entre los elementos.

Así, la cotidianidad escolar permite la existencia de múltiples posibilidades como consecuencia de las interacciones que en ella se dan. Según Maturana (1995), las dinámicas relacionales no se expresan solo hablando, sino que se viven con todo el cuerpo. Es a través de la dinámica corporal como se revela lo emocional de cada uno. De acuerdo con Moraes y Torre (1997), se trata de un "flujo permanente de las emociones que modela nuestra cotidianidad y nuestro vivir/convivir que constituye el fundamento de todo lo que realizamos. Incluso las acciones derivadas del sentipensar, recordando que cualquier hecho de la vida tiene como base fundamental lo emocional" (p. 4). Frente a lo anterior, es necesario puntualizar que en Colombia, el Ministerio de Educación Nacional [MEN] cuenta con distintos documentos en los cuales se identifican intenciones formativas desde una perspectiva holística. Estas consideraciones pueden resumirse en los propósitos de la educación física expuestos en el documento $\mathrm{N}^{\circ} 15$ (MEN, 2010), los cuales concuerdan con las ideas recientemente expuestas. Según este documento la educación física escolar debe:

"Promover el cultivo personal desde la realidad corporal del ser humano" (p. 13), es decir, los estudiantes deben ser conscientes de su corporeidad, para comprenderse a sí mismos y poder interactuar con los otros. Esto exige la formación de actitudes y modos de actuar que otorguen sentido a las prácticas, técnicas, saberes y hábitos de actividad motriz, que fomenten la cooperación y la solidaridad. Sobre la misma línea, "la educación física debe aportar a la formación del 
ser humano en el horizonte de su complejidad" (p. 14), lo cual debe permitir el desarrollo del pensamiento, la sensibilidad, la expresión y la lúdica. Asimismo "debe contribuir a la construcción de la cultura física y a la valoración de sus expresiones autóctonas. La Educación Física debe fomentar y promocionar los saberes y prácticas apoyados en la expresión motriz." (p. 14). Igualmente debe dirigir parte de sus acciones hacia la educación de la sensibilidad y el desarrollo del lenguaje corporal.

De otra parte, "debe apoyar la formación de cultura ciudadana y de valores fundamentales para la convivencia y la paz. Por su carácter vivencial” (p. 14). La educación física permite orientar la construcción de valores sociales como el respeto a la diferencia, a la libre elección y a la participación en decisiones de interés general. La experiencia de compartir exige reconocer el valor del otro, así como dar importancia a las reglas en la acción colectiva para el cuidado de lo público y construcción de acuerdos.

Finalmente, la educación física tendrá que "desarrollar una pedagogía de la inclusión de todas las personas” (p. 15). Ninguna persona puede ser excluida ni marginada dela práctica dela educación física, por cuanto esta disciplina involucra componentes fundantes de la naturaleza humana, de modo que el trabajo pedagógico dirigido a cada ser humano en particular debe adaptarse a sus necesidades. Así, las emociones, sentimientos, lenguajes, pensamientos y demás elementos de la urdimbre humana expuestos en la cotidianidad escolar parecen ser objeto de formación en la clase de educación física, toda vez que es en ella donde este marco de trabajo se hace efectivo. En consecuencia, la observación y conocimiento de dicha realidad dará lugar a identificar aquellas acciones educativas que desde el profesor disienten o responden correctamente a los planteamientos teóricos que juegan como posibles configuradores de la realidad escolar.

\section{Problema}

Como campo de conocimiento y práctica educativa, la educación física está obligada a reflexionar y actuar frente a las múltiples problemáticas que hacen presencia en sus espacios y procesos (MEN, 1999). La solución a estas dificultades suele recaer en los maestros, pues son estos los que de una u otra forma ponen en juego 
sus maneras de pensar, saber, sentir y actuar en el día a día escolar. Una de las realidades que estos maestros deben enfrentar obedece a la presencia de mujeres adolescentes en estado de gestación, razón por la cual deben emprender diversas acciones que surgen de sus percepciones, saberes y sentires.

Se sabe que las mujeres en estado de gestación presentan cambios significativos en su salud, atraviesan por distintos momentos emocionales y sobrellevan cambios físicos. Estos factores se convierten en riesgo, pues la inestabilidad emocional del momento, la falta de empoderamiento de su cuerpo y una sexualidad sin determinaciones claras influyen de forma notoria en sus maneras de enfrentar la vida. En este sentido es fundamental preguntar: ¿cómo los maestros de educación física enfrentan esta realidad? ¿Qué sucede y qué sienten las adolescentes gestantes durante las clases? Para comprender esto es necesario saber que en América Latina y el Caribe se estima que aproximadamente el 22\% de las niñas inician su vida sexual antes de cumplir los quince años. Para el caso colombiano, el estudio realizado por Noguera y Alvarado (2012) indican que el inicio de la actividad sexual en las colombianas es de 13,8 años, lo cual lleva al país a ocupar el primer lugar en la región; 90 de cada 1000 jovencitas quedan embarazadas entre los 13 y 19 años. Según Alvarado, Martínez, Banquero, Valencia y Ruiz (2007), este problema constituye la más grave problemática en salud; cada día 19 niñas menores de 15 años se convierten en madres.

La Encuesta Nacional de Demografía y Salud realizada por el Ministerio de Salud (2016) encontró que la proporción de adolescentes embarazadas se incrementó significativamente entre 1990 y 2015 al pasar de 12,8 \% a 19,5 \%. Además, de acuerdo con Profamilia (2015), entre 2008 y 2013, en Colombia nacieron en promedio 159657 hijos de madres entre 10 y 19 años. Las cifras más preocupantes se encuentran en Antioquia -595 nacimientos por cada mes-, Bogotá -578- y Santander -358-; cifras alarmantes, teniendo en cuenta que esta información corresponde a la etapa de escolarización básica de estas mujeres. El informe regional sobre desarrollo humano del Programa de Naciones Unidas para el Desarrollo (2016) afirma que Colombia ocupa el quinto lugar de la región con mayor tasa de natalidad de adolescentes y jóvenes entre los 15 y 19 años. El informe señala que Colombia requiere de alternativas para que las adolescentes en estado de gestación 
reciban una atención integral, educación y formación profesional a futuro. El inicio precoz de las relaciones sexuales, la no utilización de métodos anticonceptivos, la ruptura del grupo familiar, la falta de comunicación con los padres y la falta de procesos educativos efectivos sobre la capacidad de medir riesgos son factores que inciden negativamente en el crecimiento de estas cifras (Flórez y Soto, 2013).

Este fenómeno, que sin duda alguna desborda la capacidad de respuesta de la escuela para generar alternativas que reduzcan sus efectos, debe ser un motivo suficiente para emprender procesos de conocimiento que de alguna u otra forma aporten a la comprensión de los factores que allí se presentan. Castillo, Castro y Barría (2012) encuentran que un alto porcentaje de estudiantes embarazadas no se vinculan a la clase de educación física debido a que suelen estar llenas de temores y no logran entender lo que les pasa. Por eso, antes de buscar una estrategia o alternativa para favorecer la participación activa de las escolares gestantes, es fundamental indagar acerca de los sentires y saberes de los maestros y comprender la relevancia de sus procesos, imaginarios y de los ambientes que gestionan, pues en ellos se encuentra una ruta que conduce a la concreción de procesos efectivos, cercanos y personales que de cierto modo se apartan de los modelos de atención convencional. Es por esto que conocer el marco de acciones que los maestros configuran en su cotidianidad al momento de enfrentar estas situaciones resulta clave para pensar un escenario de posibilidades en el cual las actuaciones del docente sirvan para hacer de la clase un espacio de aprendizaje y discusión sobre realidades sociales en las que el cuerpo y sus dimensiones reconfiguran la existencia de cada sujeto.

\section{Diseño metodológico}

Esta es una investigación cualitativa-exploratoria que trabaja con registros descriptivos y entrevistas semiestructuradas. Busca interpretar lo que sienten, piensan y hacen tanto los docentes, como las estudiantes en estado de gestación en la clase de educación física. Los registros se realizaron por medio de la técnica de observación no participante (Bautista, 2011), en formatos de observación enfocados en tres aspectos de acción: actividades propuestas por el docente, comportamiento del docente y respuesta de las madres gestantes. 
Para observar los pensamientos de los actores, como los sentimientos que producían las distintas situaciones y prácticas, se diseñaron dos entrevistas semiestructuradas (Bautista, 2011), una para cada actor. Su objetivo fue identificar rasgos particulares sobre el saber-sentir-pensar de los docentes y sobre el sentir-pensar de las estudiantes. Este diseño fue validado por tres expertos en investigación cualitativa y una prueba piloto con un docente con experiencia en la inclusión de madres gestantes en sus clases y una estudiante en estado de gestación. Las preguntas giran en torno al conjunto de conocimientos, sensaciones, percepciones, emociones, problemas y actividades que se ponen en juego durante las clases. Cabe aclarar que la lectura y análisis de las primeras entrevistas condujo a nuevos interrogantes que fueron abordados en encuentros posteriores durante el trabajo de campo, es decir, desde la planeación inicial se consideró efectuar un análisis gradual de la información con el objetivo de resolver las dudas que fueron apareciendo.

Es importante señalar que, por tratarse de una investigación cualitativa-interpretativa, esta se aparta de los criterios estadísticos y focaliza su proceso en el significado de los datos. En consecuencia, la selección de los participantes se realizó considerando la relevancia de los casos y las expectativas generadas teóricamente para el trabajo de campo. Los participantes son tres docentes de educación física y tres adolescentes en estado de gestación, adscritos a tres colegios del centro del país, de los cuales dos son instituciones escolares del municipio de Soacha y la otra de la ciudad de Bogotá. Su vínculo se hizo mediante efecto bola de nieve (Bautista, 2011), lo que permitió identificar en las instituciones escolares la presencia de adolescentes en estado de gestación. Es importante mencionar que la selección de las dos primeras instituciones obedece a que el municipio de Soacha, según la Gobernación de Cundinamarca (2013), ocupa el segundo lugar de embarazos adolescentes en el departamento. Además, su proximidad territorial con Bogotá, centro urbano con mayor densidad demográfica del país, da la oportunidad de interpretar las particularidades de los contextos, motivo por el cual se incluyó una tercera institución localizada al interior de la capital. Las adolescentes vinculadas al estudio presentan embarazos normales de 24, 20 y 26 semanas de gestación y todas cursan grado décimo. Para acceder a la información, fue necesario firmar un 
consentimiento por parte de los directivos, docentes, padres de familia y estudiantes que participan de la investigación. El trabajo de campo se realizó tres veces por semana durante un periodo de cinco meses entre febrero y marzo de 2015, para un total de sesenta sesiones de observación y seis entrevistas con un promedio de 56 minutos cada una.

\section{Proceso de análisis}

Para el análisis de la información se tomaron las declaraciones de ambos actores junto con las actividades de clase y comportamientos del docente. Esta información fue analizada en tres momentos: el primero corresponde a la codificación axial de los archivos (Coffey \& Atkinson, 2003). En esta se identifican aquellas declaraciones y registros asociados al hacer, sentir y pensar de los participantes a partir de lectura analítica. Luego se clasifica la información en una matriz de doble entrada. En el segundo momento se selecciona la información considerando los aspectos característicos de las declaraciones y observaciones, es decir, se identifican los elementos emergentes, su significado y el vínculo que guardan con los desarrollos conceptuales de la investigación. Finalmente, la interpretación de la información se realizó a través del principio dialógico propuesto por Morin (2009) y según un nuevo proceso de codificación y agrupamiento, con el propósito de construir una red de sentido que permite entablar un diálogo entre el sentir, pensar y hacer de los actores con los aspectos emergentes identificados. En cuanto a la citación de las evidencias empíricas y su relación con las interpretaciones que se hacen de ellas, se ha establecido el siguiente esquema de identificación: $\mathrm{do}=$ docente; $\mathrm{e}=$ entrevista; es= estudiante; $\mathrm{y}$ fo = ficha de observación. Estas referencias van acompañadas de un consecutivo con el cual se identifica el número asignado a los participantes, entrevistas y fichas de observación.

\section{Resultados y discusión}

El profesor ofrece atención a la madre gestante según las condiciones físicas que ella presenta. Su pensar se configura en virtud de las recomendaciones médicas y de los discursos de la salud que hacen parte de la formación disciplinar. "Me hacía caminar alrededor de la cancha. Decía que los médicos recomendaban pequeñas caminatas diarias para fortalecer los músculos abdominales y 
la región pélvica, que son muy importantes en el parto" (es3.e2). Algunas de las decisiones del profesor provienen de su conocimiento de las estructuras corporales, razón por la cual el cuerpo biológico cobra mayor importancia, pues se trata de la presencia material del sujeto en el mundo. Sin embargo, este discurso no trasciende las posibilidades de lo orgánico y delimita lo corporal a lo funcional: "una madre gestante debe realizar ejercicios de fortalecimiento pélvico y ejercicios de respiración. Esto es importante, pues a la hora del parto tendrá fortalecida la pelvis y un mejor manejo de la respiración" (do3.e2). Así, las estudiantes deben participar de sus clases "haciendo las actividades iniciales de clase, estiramiento, movilidad articular, y luego con pausas; caminatas más o menos de cinco minutos, por pausas de descanso, y de acuerdo con lo que recomienden los médicos en el curso psicoprofiláctico" (do2.e1).

Para Araujo (2013), "los sentimientos son el ingrediente básico de todo posible conocimiento capaz de trasformar individuos y sociedades" (p. 133). Sin embargo, buena parte del trabajo con las madres gestantes se enfoca en fortalecer lo físico pensando en la prevención: "se evita incluirlas en las actividades deportivas o de alta exigencia física y motriz" (fog), en tanto se puede causar daño. En este sentido, el docente opta por asignar otro tipo de tareas entre las que se encuentran los estiramientos, los trabajos escritos o la supervisión a sus compañeros: "a veces realizaba circuitos de estiramientos en los que yo participaba, en muchas ocasiones sentía que realizaba una clase específica para mí, aunque a veces me separaba del grupo" (es3.e2).

Frente a lo anterior, es evidente que los docentes observados reconocen el cuerpo femenino y sus necesidades fisiológicas y anatómicas con cierta prevención. Aunque conocen los cambios por los que transitan las gestantes, estas dinámicas que paulatinamente van transformando la imagen y estructuras corporales de las estudiantes influyen sobre las decisiones y actuaciones del profesor. Se dejan a un lado los procesos lúdicos y socioafectivos que aportan emocionalidad a la escolarización, para dar mayor importancia a la madurez física que garantiza el desarrollo normal de los procesos orgánicos del embarazo: "la madurez es muy importante sobre todo en estos casos. Por ello se debe tener en cuenta como primera medida eso, que son niñas que no han madurado" (do1.e1). Así, los riesgos que enfrentan las adolescentes afectan las emociones de las 
estudiantes, pues en distintos momentos manifestaron sus deseos de participar, así como su malestar al "separarlas para hacer otras tareas" (fo4). No obstante, el profesor presta especial cuidado a la madre, a expensas de no excluirla de las demás actividades.

$\mathrm{Al}$ conocer de la existencia de otro ser dentro del cuerpo de la estudiante y de los cambios que se presentan en ella, los docentes se informan con el propósito de tomar decisiones que disminuyan los riesgos, "usando la internet y textos referentes al tema" (do2.e2). Sin embargo, estos procesos siguen enmarcados en los enfoques higienistas, es decir, su preocupación gira en torno al cuidado del cuerpo y la salud, mientras desconocen los factores subjetivos y sociales presentes allí. Este panorama no deja de ser preocupante, pues nuevamente los docentes asumen los aspectos biofísicos de la madre y su bebé como los más importantes y prioritarios. A pesar de informarse y reconocer los riesgos en el embarazo de sus estudiantes, no identifican claramente la mayor parte de las dificultades que estas pueden presentar, como "hipertensión, poca ganancia de peso, anemia, parto pretérmino, deserción escolar, alteraciones en los procesos familiares y alteraciones en el desempeño del rol materno" (Noguera \& Alvarado, 2012, p. 152), sin olvidar los estados de ansiedad o depresión. En este sentido, los factores mencionados pueden afectar el desempeño, motivación y participación de las gestantes.

Es evidente el temor al daño que puede causar el maestro, razón por la cual pierde de vista la complejidad de la situación y retorna a las propuestas enfocadas en la ejercitación y la prevención de riesgos proyectadas al parto.

La adolecente que está en estado de gestación debe participar activamente. Obviamente uno como docente debe saber que ella tiene algunas restricciones por su estado, lo más importante es indagar qué ejercicios se pueden hacer, ya que todos los ejercicios no son buenos para el niño (do1.e3).

El profesor propicia espacios de trabajo en los cuales genera acciones conducentes a la preservación de la seguridad del niño y la madre. En consonancia con lo anterior, las estudiantes asumen que las decisiones del docente son coherentes con sus necesidades particulares, pues el tratamiento diferenciado se considera como hecho de conocimiento al asignar las tareas de la clase. Una de las 
estudiantes manifiesta: "me siento segura, porque sé que el profesor no me va a poner ejercicios de tanta fuerza que me afecten a mí y al bebé" (es3.e3). Entonces, el saber disciplinar del docente aporta al buen estado de la madre, pero no desborda su dimensión biológica funcional.

Lo que yo siempre hago con ellas es especialmente caminatas, la caminata con pausas. Ellas caminan cinco minutos, descansan cinco o seis minutos, y luego vuelven a caminar, y participan de los ejercicios, como ya había dicho, estiramientos y movilidad articular de forma muy suave (es2.e1).

Ante la evidente preocupación del docente por integrar en el desarrollo de sus sesiones y programas de aula diferentes actividades y ejercicios de apoyo al desarrollo del embarazo, es posible decir que el saber-sentir del maestro apunta a la construcción de una consciencia sobre el cuidado del cuerpo que permite a la madre adquirir conocimientos sobre las necesidades de su hijo y de sí misma.

Para la adolecente en estado de gestación, estas actuaciones del maestro muestran el grado de preocupación que tiene ante sus necesidades. Él trata de comprender con la razón y la emoción lo que sienten las gestantes: "Siento apoyo. Cuando me manda a caminar me dice que no me esfuerce tanto, porque esto le hace daño al bebé" (es1.e3). Sin embargo, dicha preocupación no está claramente definida cuando se trata de aspectos socioculturales. Para Espinosa (2014), "un proceso educativo 'sentipensante' implica aprender a 'sentir al otro'; reconocer la dignidad intrínseca e igualdad sin distinción entre personas” (p. 100). Según las madres gestantes, las actividades propuestas por el maestro procuran protegerles de accidentes. No obstante, estas decisiones no son suficientes a la hora de generar estrategias de formación integral, pues la condición biofísica concentra la mayor parte de las intenciones: "pienso que los ejercicios son muy chéveres, el profe me pone a caminar, mientras mis compañeros hacen los ejercicios que él les diga" (es3.e2).

Frente a lo anterior, se puede señalar que la actividad física ha sido el medio de acción, participación e inclusión; consideración tradicional de la educación física que desconoce múltiples factores socioculturales, estructurales y contingentes que se manifiestan en la cotidianidad escolar. Sin embargo, las percepciones que 
las gestantes tienen de sí mismas y de las actividades propuestas por los docentes las conduce a cuestionar y reflexionar sobre sus posibilidades corporales y las relaciones que ellas pueden establecer con el contexto y con los demás: "también hay momentos en los que me pregunta sobre cómo me siento para realizar las actividades, sobre todo en las que se compite o se necesita ser veloz, fuerte o hábil” (es1.e2). En este sentido, el maestro recurre a las sensaciones y estados de ánimo de la madre para definir su participación en algunas actividades, especialmente las motrices. Así se ratifica la importancia del énfasis en el dominio físico-corporal, pero con una significativa diferencia: en esta ocasión se acude a las sensaciones tanto del maestro como de las estudiantes para tomar las decisiones de los procesos de la clase, lo cual supera en cierto grado la marcada importancia que se le ha otorgado a la biomecánica del cuerpo. Esto hace una evidente transición hacia lo emotivo, sensitivo y subjetivo. $\mathrm{Al}$ cuestionar los beneficios o perjuicios de las actividades mediante las emociones, las estudiantes señalan que se trata de algo relativo: "a veces no me gusta la clase porque no puedo hacer nada de lo que yo quiero hacer, y a la vez, la clase es buena para el bebé, ya que hacer ejercicios es bueno" (es2.e3).

En diversos momentos fueron las madres gestantes quienes solicitaron un trato diferencial dadas sus propias condiciones, hechos que pueden conducir a creer que dichas peticiones obedecen a la lectura que ellas hacen de sus propias sensaciones corporales y anímicas, es decir, el diálogo con sí mismas puede constituir un punto de referencia para la reflexión pedagógica de cualquier maestro, pues las decisiones de los docentes frente a este tipo de hechos pueden generar dos tipos de procesos: a) aislar a la madre para darle un trato diferencial atendiendo a las recomendaciones médicas o b) modificar la planeación curricular para responder a las necesidades colectivas, evitando la exclusión, el rechazo, el aislamiento y la selectividad en las actividades, especialmente aquellas relacionadas con las destrezas motrices y el ímpetu físico.

En este diálogo de razones afirmativas y contradictorias sobre las actuaciones y sentires de los maestros, ellos asumen que los procesos de inclusión y formación de las madres gestantes en clase de educación física deben contemplar los siguientes aspectos:

- Conciencia del estado en el que se encuentran. 
- Reconocimiento de la existencia y presencia de otro incorporado -ente en gestación-.

- Conocimiento sobre los procesos y métodos de cuidado.

- Realización de actividades de apoyo al proceso de gestación.

En este sentido, el saber-pensar del docente y el sentir de la estudiante están circunscritos a una temporalidad propia del momento, es decir, los factores contextuales -cambios en la naturaleza del cuerpo- ejercen presión sobre las decisiones tanto de uno como de otro. De acuerdo con Araujo (2013), "hoy, mejor que nunca, sabemos gracias a los aportes de la neurociencia que somos una unidad sentipensante por naturaleza" (p. 133). En este sentido, las circunstancias que se presentan conducen a la toma de nuevas decisiones, intuiciones y sentimientos que se producen al relacionarse con la gestante, es decir, con su estado. En otras palabras, lo que se siente depende de las siguientes relaciones:

- Docente-estudiante en gestación, en la que la madre experimenta nuevas sensaciones de su propio cuerpo, así como cambios en las estructuras corporales y, en consecuencia, modificación de la representación e imagen corporal. En cuanto al profesor, él construye una nueva representación del cuerpo de la estudiante, asociada a las posibilidades físico-motrices.

- Docente-ente gestado, en la que el profesor desconoce las características del desarrollo del ente gestado, motivo por el cual procede con cautela a través de la madre.

- Estudiante en gestación-ente gestado, en la que la madre identifica las limitantes de su cuerpo y transita por el reconocimiento de un nuevo ente con cierto grado de autonomía integrado a su cuerpo con el cual confronta sus propios deseos y miedos.

Estas nuevas configuraciones del pensar docente y del sentir de la estudiante no son ingenuas e imprevistas, pues como se puede evidenciar en sus declaraciones, los maestros asumen el estado de gestación como una contingencia que requiere de un reacomodamiento de las condiciones del sujeto y ante las cuales se deben prever nuevas acciones. $\mathrm{Al}$ respecto, uno de los maestros indica: 
Me siento capacitado para incluir a las estudiantes. Lo he hecho en mi experiencia de vida; al principio las aislaba, yo las dejaba quieticas... allá, pero con el tiempo noté que estaban como tristes. No estaban bien, entonces, decidí dejarlas participar y hasta ahora no he tenido ningún inconveniente (do2.e2).

Frente a esto, es evidente el interés, seguridad y enfoque emocional que el profesor va construyendo. A medida que se enfrenta a la situación, propone en sus actividades la participación de la estudiante. Tales decisiones van emergiendo en la medida que el contexto lo confronta con su propio pensar y sentir. Para Damasio (2000), la razón - que para el caso se aquí se analiza corresponde a la didáctica de la clase- precisa de las emociones porque la capacidad de control de la razón es a menudo modesta e incipiente. De este modo, los sentimientos manifiestos en las estudiantes comunican con los sentimientos y reflexiones del maestro, motivo por el cual pueden operar como fuerzas activadoras y dinamizadoras de las clases, es decir, las emociones emergentes propias en los distintos momentos y sujetos dela clase pueden conducir a incluir o desestimar procesos y actividades. En otros casos la situación es distinta. Según las experiencias de las gestantes, las actividades están centradas en lo motor: "caminar y caminar... eso es lo que hago en la clase, y entregarle trabajos al profesor si me ha dejado alguno, y me dice que me haga en el sol" (es1.e3). La estudiante hace presencia en la clase, pero se le excluye por su estado. El docente en su desconocimiento improvisa la decisión y reemplaza la participación por actividades en las que el vínculo expresivo y motor con los demás estudiantes desaparece.

\section{Conclusiones}

El estudio identifica en los docentes observados algunos sentidos prácticos de la educación física configurados desde el discurso de la salud, la prevención y el desarrollo motor. De acuerdo con esto, es importante señalar el marcado énfasis sobre la dimensión biofísica de las estudiantes, razón por la cual se abre la discusión frente al conjunto de aspectos sociales, culturales, económicos y otros que no son relevantes en los procesos educativos que los profesores emprenden en sus clases.

Por otra parte, es notorio el interés por el cuidado de las adolescentes a partir de las acciones y decisiones de los profesores 
en los que la emoción y el saber se encuentran cruzados. Llama la atención el grado de selectividad al momento de asignar las tareas, pues en sus acciones prevalece la extraña necesidad de apartar a las estudiantes. Con justa razón, los temores y desconocimiento del docente, junto con el riesgo al que se puede exponer la gestante por una mala decisión pedagógica, impiden que el maestro les involucre en otras actividades, particularmente aquellas de carácter colectivo $\mathrm{u}$ otras en las que el contacto y la intensidad de los movimientos es notorio. Sin embargo, dicha razón deja en entredicho las tesis sobre la diversidad de actividades y posibilidades didácticas que la educación física profesa y pone sobre la mesa la inocencia excluyente que puede generar un trato diferencial en las personas al punto de hacerlas sentir excluidas o limitadas en sus posibilidades y capacidades.

En diversas observaciones, al igual que en múltiples declaraciones, tanto de las madres gestantes como de los profesores, aparece una constante preocupación por el daño físico al cuerpo de la gestante. No obstante y sin ánimo de opacar la labor del maestro, es evidente la poca o nula importancia de la vida social y emocional de la madre al interior de la clase. En los discursos no se pueden evidenciar declaraciones en las cuales los aspectos socioemotivos tengan el mismo peso que tiene el temor al daño o el cuidado del cuerpo. De igual forma, parece que las constantes alusiones de las gestantes frente a su inclusión en la clase reflejan una manera particular de sentir asociada al menosprecio de su condición física, concretamente sobre su desempeño motor. En consecuencia, la imagen corporal y el desenvolvimiento motor de la madre gestante produce en ella misma un conjunto de sentimientos que se traducen en formas específicas de hacer que no necesariamente responden a los estereotipos técnico-corporales, sino a un reclamo por el lugar que cada sujeto ocupa en la vida social de la clase.

Es importante destacar el interés por adquirir conocimiento asociado al cuidado de la persona, pues como se pudo evidenciar, las estudiantes apropian paulatinamente saberes sobre el cuidado del cuerpoy los peligros que pueden correr en la clase. Sin embargo, dicho grado de riesgo se encuentra asociado a las dinámicas imperantes de la educación física, pues en las instituciones observadas el desempeño físico y el desarrollo motor han constituido el eje central de las propuestas educativas. En este sentido, la educación 
física precisa dialogar con otros esquemas educativos que también aportan a la formación integral que la disciplina profesa. Si bien los riesgos y los aspectos emergentes hacen que los docentes se inclinen por cambiar su forma de asumir la clase, es evidente que una buena parte de estos riesgos se genera por las formas de pensar y practicar la educación física escolar.

\section{Referencias}

Alvarado, R., Martínez, O., Banquero, M., Valencia, C., \& Ruiz, A. (2007). Problemática en torno a adolescentes de 14 a 19 años en Nocaima (Cundinamarca). Revista de ciencias de la Salud, 5(1), 40-52.

Araujo, J. (2013). Aproximación hacia una educación sentipensante. Colección de Filosofía de la Educación, 14, 129-140.

Bautista, P. (2011). Proceso de la investigacion cualitativa. Bogotá: El Manual Moderno

Castillo, A., Castro, M., \& Barría, A. (2012). Preparación que siente tener el profesorado de Educación Física respecto a la realización de clases a alumnas embarazadas y la participación de estas en ellas. Efdeportes revista digital. (168). Recuperado de http://www.efdeportes.com/ efd168/educacion-fisica-clases-a-alumnas-embarazadas.htm

Coffey, A., \& Atkinson, P. (2003). Encontrar el sentido a los datos cualitativos. Medellín: Universidad de Antioquia.

Damasio, A. (2000). Sentir lo que sucede. Santiago de Chile: Andrés Bello.

Espinosa, D. (2014). Una escuela sentipensante para el reconocimiento y práctica de los derechos humanos. Rastros Rostros, 16(30), 95-104.

Flórez, E., \& Soto, V. (2013). Factores protectores y factores de riesgo del embarazo adolescente en Colombia. Encuesta Nacional de Demografía y Salud. ENDS. Bogotá: Profamilia

Gobernación de Cundinamarca. (2013). Anuario estadístico de Cundinamarca. Bogotá: Gobernación de Cundinamarca.

Maturana, H. (1995). La realidad: ¿objetiva o construida? II. Fundamentos biológicos del conocimiento. Barcelona: Anthropos.

Maturana, H., \& Varela, F. (1999). El árbol del conocimiento. Las bases biológicas del entendimiento humano. Santiago de Chile: Editorial Universitaria. 
Ministerio de Educación Nacional de Colombia. (1999). Lineamientos curriculares en educación física. Bogotá: MEN.

Ministerio de Educación Nacional de Colombia. (2010). Orientaciones pedagógicas para la educación física, recreación y deporte. Bogotá: MEN.

Ministerio de Salud de Colombia. (2016). Informe nacional de demografía y salud. Bogotá: Minsalud.

Moraes, M., \& Torre de la, S. (2002). Sentipensar bajo la mirada autopoiética, o cómo reencantar creativamente la educación. Revista creatividad y sociedad, 2, 41-56.

Morin, E. (2009). Introducción al pensamiento complejo. Madrid: Gedisa.

Noguera, N., \& Alvarado, H. (2012). Embarazo en adolescentes: una mirada desde el cuidado de enfermería. Revista colombiana de enfermería, 7, 151-160.

Profamilia. (2015). Encuesta Nacional de Demografía y Salud ENDS. Bogotá: Profamilia

Programa de Naciones Unidas para el Desarrollo. (2016). Informe regional sobre desarrollo humano para América Latina y el Caribe. Progreso multidimensional: bienestar más allá del ingreso. New York: Phoenix Design.

Torre de la, S. (2011). Didáctica e Innovación Educativa. Barcelona: Universidad de Barcelona. 